COMENTARIO EDITORIAL

\title{
Barreras para la implementación de la prevención en la práctica clínica
}

\begin{abstract}
Introducción
Los beneficios sociales de incorporar la prevención a la práctica médica se han vuelto tangibles en los últimos 40 años. En la mayoría de los casos, esto se debió a intervenciones activas tales como la masiva implementación de inmunizaciones y prácticas de diagnóstico temprano, concentradas estas últimas, en la prevención del cáncer y de las enfermedades cardiovasculares. Como consecuencia de ello, la incidencia de poliomielitis en EE.UU. se redujo de 18.300 casos en 1954 a sólo 3 casos en 1983, con tendencias similares en otras enfermedades infecciosas prevenibles como el sarampión, rubéola, difteria, crup, Haemophilus influenzae, etc. También en los últimos 30 años, debido a la mejoría en el diagnóstico y manejo de la hipertensión arterial, el control del tabaquismo, la hipercolesterolemia, el sedentarismo y otros factores de riesgo cardiovascular, algunos países desarrollados como EE.UU., Canadá y Reino Unido redujeron 50\% la mortalidad ajustada por edad para accidentes cerebro-vasculares, aproximadamente $30 \%$ la mortalidad por complicaciones de la enfermedad coronaria, se produjo además una dramática reducción de la incidencia de morbimortalidad por cáncer de cuello invasor o de trastornos cognitivos por hipotiroidismo o fenilcetonuria en los niños. ${ }^{1}$
\end{abstract}

A pesar de estos logros, el mayor desafío actual de la prevención consiste en poder modificar algunos estilos de vida que aumentan el riesgo de enfermedades 0 accidentes y que son potencialmente prevenibles a través de cambios en hábitos y prácticas personales ${ }^{2}$. En efecto, al menos $50 \%$ de las muertes pueden ser atribuidas, en gran medida, a conductas personales tales como el tabaquismo, el tipo de dieta, la falta de empleo de cinturón de seguridad, el consumo excesivo de alcohol, las drogas ilícitas, el sedentarismo y las prácticas sexuales inseguras, entre otras. ${ }^{3-4}$. De hecho, el tabaco es responsable de una de cada 5 muertes ocurridas por cáncer y enfermedades cardiovasculares ${ }^{5}$.

Se han identificado numerosas barreras relacionadas con los médicos y demás profesionales de la salud, con los pacientes y con el sistema de salud, que explican la falta de cumplimiento de las acciones preventivas ${ }^{6}$. En la siguiente sección, nos extenderemos en ciertas consideraciones sobre las barreras que dependen de los prestadores de salud y de los pacientes/usuarios, para luego profundizar un poco más en aquéllas que están fundamentalmente relacionadas con las características del sistema de salud en donde se desenvuelven los prestadores y los consumidores de servicios clínicos.

Barreras que impiden la provisión de servicios preventivos dependientes de los prestadores y de los pacientes/usuarios

Las barreras relacionadas con los médicos incluyen la falta de entrenamiento en prevención, un sesgo hacia la acción de curar y una sobreestimación del valor de los diagnósticos y tratamientos complejos. ${ }^{7}$ También se ha identificado la falta de tiempo en la consulta médica, la insatisfacción, la falta de incentivos económicos, y la falta de acuerdo y uniformidad de las guías preventivas. 8 Por otro lado, las barreras que dependen de los pacientes tienen que ver con factores culturales basados en valores, actitudes y creencias sobre el significado de la prevención y qué cosas vale la pena prevenir, así como mensajes contradictorios de los prestadores o los seguros acerca de las acciones apropiadas en prevención clínica. En numerosas ocasiones, los pacientes suelen demandar "chequeos completos" para detectar cualquier problema de salud que los pueda estar afectando, a la vez que desconocen cuáles medidas han demostrado ser efectivas para disminuir la morbimortalidad. También pueden sentir que las medidas preventivas interfieren con su actividad usual, como por ejemplo colocarse casco cuando andan en bicicleta ${ }^{9}$. La consulta episódica y la falta de cuidados continuos, además de los inconvenientes atribuibles a las pruebas diagnósticas, el temor a los resultados positivos, los costos de la práctica, o las consecuencias del diagnóstico en el caso de las intervenciones de diagnóstico temprano, son otras razones que conspiran contra la mayor extensión y efectividad de las acciones preventivas. ${ }^{10-11}$ Varias estrategias han sido ensayadas para mejorar la provisión de servicios preventivos en atención primaria incluyendo educación ${ }^{12}$, feed-back 13 , rediseño de sistemas administrativos, e incentivos financieros. ${ }^{14-15}$ En un estudio realizado en un centro ambulatorio perteneciente a un hospital académico, nosotros demostramos que la educación a los prestadores y usuarios, junto a auditoría y feed-back a los prestadores, mejoraban la efectividad de ciertas intervenciones preventivas seleccionadas. ${ }^{17}$

Barreras que impiden la provisión de servicios preventivos dependientes del sistema de Salud

Si bien la adherencia de los médicos y de los pacientes son fuertes determinantes del cumplimiento de las recomendaciones en prevención clínica, existen factores sistémicos que definen el marco político, social y económico de la prevención. Es posible identificar 3 niveles de ordenamiento de la estructura del sector salud donde estas barreras pueden aparecer: a nivel macro, la definición de políticas públicas; a nivel intermedio, las características del sistema de atención médica; y a nivel micro, las características e incentivos de los prestadores.

\section{Políticas públicas pobres}

El gasto público estatal y en particular el gasto social, en el cual está incluído salud, es tal vez el indicador más contundente de inversión social y humana que un país puede demostrar. El porcentaje del gasto total en salud financiado con fondos públicos (\% gasto público en salud, \% GPS), a diferencia del gasto total o el porcentaje del PBI que se gasta en salud, es por definición un gasto redistributivo y solidario. Indica cuán equitativa es la asignación de recursos en este área. Las políticas de prevención, en especial las de prevención primaria como inmunizaciones, cambio de hábitos y de conductas de riesgo, etc., pero también las de prevención secundaria como el diagnóstico temprano, presentan lo que los economistas llaman externalidades. Esto quiere decir que lo que se hace con unos afecta también a otros. Las vacunas, por ejemplo, no sólo protegen a quien se la aplica sino también a los contactos. Por lo tanto, las políticas públicas son decisivas porque se trata de preservar el bien común. En la mayoría de los países desarrollados a excepción de Estados Unidos y Alemania, el \%GPS supera el $70 \%$ y no casualmente esos países son los que presentan los indicadores de efectividad de programas de prevención primaria y secundaria más altos, así como los sistemas de salud más orientados a la atención primaria. ${ }^{17-18}$ En la Argentina, por el contrario, el \%GPS real es sólo del 22\% - sin incluír el gasto de las Obras Sociales, que dada la fragmentación y las características que han adquirido últimamente, difícilmente pueda considerarse gasto solidario-, cifra que explica con suficiente elocuencia los magros resultados alcanzados que podemos exhibir en nuestro país.

\section{Sistema de atención médica fragmentado e incordinado}

$\mathrm{Si}$ acordamos con la existencia de externalidades implícitas en cualquier intervención preventiva, un sistema fragmentado ofrece mayores dificultades para coordinar dichas acciones. Mas aún si el sistema prevaleciente tiene financiamiento privado o mixto. En los países con sistemas nacionales de salud como Reino Unido y España, o con sistemas provinciales como Canadá, en los cuales hay casi un único financiador que es el Estado, la implementación de políticas de prevención 
depende de decisiones en un solo nivel. En países con sistemas fragmentados basados en la seguridad social y con fuerte financiamiento privado, como Holanda y Alemania, la coordinación de políticas depende del rol regulador y fiscalizador del Estado. Otro ejemplo es Estados Unidos, donde ccmo consecuencia de sus propios valores sociales en los cuáles es preferible la "autorregulación" del sector privado a la intromisión del Estado, y de la creciente penetración de los seguros comerciales en el mercado de la salud (Sistemas prepagos con fines de lucro), las mismas organizaciones crearon el comité nacional de aseguramiento de la calidad (NCQA) como manera de contrarrestar la influencia regulatoria federal. Este comité creó instrumentos de medición de resultados como el HEDIS (health employment data and information set) para monitorear la calidad de las distintas prepagas y emitir informes públicos. Muchos de las variables medidas tienen que ver con acciones preventivas primarias, secundarias y terciarias, como la tasa de recién nacidos de bajo peso, ciertas prácticas preventivas seleccionadas, niveles de hemoglobina glucosilada en diabéticos, internaciones en asmáticos, etc. ${ }^{19}$ Lo sucedido en Estados Unidos cobra especial relevancia en nuestro país donde a pesar de la creciente influencia de organizaciones de gestión y en muchos casos de financiamiento privado luego de la desregulación de las obras sociales, no existe ni regulación estatal ni autorregulación privada. Entonces, ¿cuál es el incentivo que tienen las obras sociales o los seguros privados para ofrecer y extender las prácticas preventivas para su población?. Si bien es cierto que las organizaciones privadas sin fines de lucro tienen una misión diferente de aquéllas con afan de lucro y que la prevención es parte de dicha misión 20, el argumento de que es mejor prevenir antes que curar, se diluye cuando por efecto de la "libre elección" del seguro, el sujeto sobre el cual se invirtió en prevención durante años o décadas, pertenece ahora a otro seguro que está "aprovechando" en su propio beneficio, su mejor estado de salud. Esta suerte de subsidios cruzados entre diferentes seguros oscurecen la implementación de acciones preventivas, más allá de la utilización de las mismas como estrategia de marketing para captar usuarios más saludables. En Argentina, la única manera de asegurar el cumplimiento de las prácticas preventivas, sobre todo en el sector privado, es por medio de una fuerte regulación del Estado, el que debe definir cuáles son las intervenciones que debieran ser cubiertas obligatoriamente.

\section{Características del sistema de pago de la actividad médica}

Finalmente, pero no menos importantes, son los aspectos inherentes a la forma de compensación de la actividad médica. El sistema de pago por prestación o acto médico, que sigue prevaleciendo en países como Estados Unidos o la Argentina (aunque ya en retirada), sólo compensa lo que se hace y a quien lo hace y por lo tanto no ofrece ningún incentivo para la integración de los distintos niveles donde se brindan los servicios. Un caso extremo es el de un hospital comunitario en Idaho, Estados Unidos, que implementó un programa de prevención de accidentes de bicicleta con el uso de casco y como consecuencia de esto vió reducida la facturación anual de su guardia externa en $\$ 150.000$. En la misma línea, la implementación de un programa de educación a los padres de niños asmáticos en otra institución redujo las consultas a la guardia y las internaciones de dicha población. Si cada nivel de atención es remunerado por los servicios que brinda, el hospital de Idaho no tiene razones para implementar su programa de prevención. de accidentes ni el otro hospital de implementar la educación a los asmáticos 21 . Mas aún, la implementación de programas preventivos en este contexto puede ser autodestructiva. Por el contrario, los sistemas de pago capitado, debido a que compensan la actividad médica a un valor constante que es independiente de los servicios que realmente son utilizados, ofrece un incentivo claro para la integración de servicios y por lo tanto para la ejecución de acciones preventivas.

\section{Conclusiones}

La efectividad en la implementación de servicios preventivos guarda relación con una multiplicidad de variables. Si bien la literatura médica y fundamentalmente las revistas clínicas, han estudiado más aquéllas relacionadas con los usuarios y los médicos (mucho más estos últimos), los factores sistémicos vinculados al sistema de salud donde tienen lugar todas las interacciones clínicas, son los que probablemente más incidan en los resultados. Si bien a todo nivel se puede hacer prevención y los médicos de atención primaria tienen aún muchas más oportunidades de realizarla, en cada consulta y con cada paciente, dichos factores sistémicos son insoslayables cuando nuestra mirada se extiende desde el árbol hacia el bosque.

\title{
Jefe. Unidad de Medicina Familiar y Preventiva. Hospital Italiano de Buenos Aires.
}

\author{
Referencias \\ 1. U.S Preventive Services Task Force. Guide to clinical preventive services, 2 nd ed. Baltimore. Williams and Wilkins, 1996. \\ 2. U.S Preventive Services Task Force. Guide to clinical preventive services, 2 nd ed. Baltimore. Williams \& Wilkins, 1996. \\ 3. Wechsler H, Levine S, Idelson RK, Rohman M, Taylor J0. The physician's role in health promotion- a survey of primary care practitioners. N Eng J Med 1983: $308: 97-100$. \\ 4. Schwartz JS, Lewis CE, Clancy C et al. Internists'practices in health promotion and disease prevention. Ann Int Med 1991; 114: 46-48. \\ 5. Centers for Disease Control. Cigarrete smoking-attributable mortality and years of potential life lost-United States, 1990. MMWR 1993:42-645:649. \\ 6. Stephen J, Zyzanski Ph D, Kurt C, Stange MD, PhD; Kelly R. Family Physician'disagreements with the US Preventive Service Task force recomendations. J Fam Pract. 1994:39:140-147. \\ 7. Weingarten SR, Stone E, Green. A study of patient satisfaction and adherence to preventive care practice guidelines. Am J Med.1995: 99: 590-6. \\ 8. Luckmann R, Melville SK. Periodic health evaluation of adults: a survey af family physicians.J Fam Pract. 1995; 40: 547-54. \\ 9. Thompson RS, Taplin SH, McAfee. Primary and secondary prevention services in clinical practice. JAMA 1995; 273: 1130-5. \\ 10. Mc Vea K: Crabtree BF, Medder JD et al. An ounce of precvention? Evaluation of the "Put Prevention into Practice" program. J Fam pract, 1996:43:4, 361-369. \\ 11. Facilitating prevention in primary care. BMJ.1984: 289:1585-87 \\ 12. Fullard E, Fowler G, Gray M. Promoting prevention in primary care: controlles trial of low technology, low cost approach. BMJ 1987; 294:1080-82. \\ 13. Mc Phee SJ, Bird JA, Fordham D, Rodwick JE et al. Promoting cancer prevention activities by primary care physicians: results of a randomized, controlled trial. JAMA 1991:266:358-64. \\ 14. Davidson RA, fletcher SW, Retchin S, Duh S. A nurse initiated reminder system for the periodic health examination: implementation and evaluation. Arch Intern Med. 1992: 144: $2167-70$. \\ 15. Atri J, Falshaw M, Livingstone et al. A Fair shares in health care? Ethnic and socioeconomic influences on recording of preventive care in selected inner London general practices. \\ Healthy eastenders project. BMJ, 1996; 312: 614-617. \\ 16. Rubinstein A, Kopitowski K y col. "Evaluation of Education vs. Education, Audit and feed-back in the Provider Compliance with Preventive Services in the Primary Care Setting". Jour- \\ nal of General Internal Medicine. 1996:Vol 11 Supp 1: 128 (abs). \\ 17. OCDE 1998 \\ 18. Starfield B. Primary Care. Balancing health needs, services, and technology. Oxford University Press. New York Oxford. 1998 \\ 19. Bodenheimer T. The american Health Care System. The movement for improved quality in health care. NEJM 1999:340:488-492. \\ 20. Nudelman P. The "value added" of not-for-profit health plans. NEJM 1996;334: 1057-1059 \\ 21. Berwick DM. Quality of Health Care. Part 5: Payment by Capitation and the Quality of Care. N Eng J Med 1996;335:1227-1231
}

